\title{
Astronomical Research and Outreach in Georgia
}

\section{Todua}

Address: E.Kharadze Abastumani Astrophysical Observatory at Ilia State University, Georgi E-mail:mayatodua@iliauni.edu.ge

\begin{abstract}
Interest in astronomy has been significantly increased in Georgia. As a result, the development of astronomical research, education and public outreach are in progress. In Abastumani Astrophysical Observatory - a research institution at Ilia State University - the observational and theoretical studies comprise a wide range of topics in astronomy and adjacent fields: solar system bodies, solar physics, stellar and extragalactic astronomy, theoretical astrophysics, cosmology, atmospheric and near space physics. Georgian scientists are involved in wide international collaboration and participate in a number of networks and projects. Astronomical education at bachelor, master and doctoral levels are held at Ilia University. PhD programs are also offered at other universities. In 2018, under the financial support of the World Bank and Georgian government, the renovation of Abastumani Observatory has been started. International conferences and workshops have been carried out in Georgia. Excursions at the Observatory and public lectures in astronomy throughout the country are carried out. Amateur astronomers organize astronomical events. A private observatory is under construction near Tbilisi. All these puts better perspective for future development of astronomy in Georgia.
\end{abstract}

\section{Introduction}

The center of astronomical research in Georgia is E. Kharadze Abastumani Astrophysical Observatory (ABAO). Found in 1932, it was the first astronomical observatory built in mountainous region in former USSR, at the altitude of 1650 meters a.s.l. The location for the observatory was selected due to excellent atmospheric conditions for astronomical observations.

The first major telescope of the Observatory was installed in 1937. It was the 40-cm refractor produced by the company "Carl Zeiss". Since then this telescope was actively used to observe the Moon, planets and minor solar system bodies, variable stars. It had operated for almost 70 years. In total, 14 telescopes had been installed in Abastumany, main of which are the $125-\mathrm{cm}$ reflector, $70-\mathrm{cm}$ Meniscus telescope and $53-\mathrm{cm}$ solar coronagraph. Along with astronomical research, study of the Earth's atmosphere also started in 1930-ies. Several telescopes are operational today, as well as the instruments for atmospheric research. 
Research fields in ABAO comprise all major branches of astronomy, astrophysics, cosmology and atmospheric physics. Various objects were observed and studied in ABAO during its history: the Moon and solar system bodies (planets, minor planets, comets, asteroids), the Sun, variable stars of different types, nebulae, multiple stars and stellar clusters, exstragalactic objects, early Universe. Number of discoveries and achievements are made (including supernova, novae, comets, etc.). Rich observational material was accumulated in the library of astronomical plates and films. Stellar catalogues, galactic atlases and lunar maps were made. Most of the catalogues are stored in the SAO database. Atmospheric studies are one of the important fields in ABAO, systematic observations of which started in 1957.

Until 2007 the Observatory was an institute of Georgian National Academy Of Sciences. In 2007 it was integrated with Ilia State University, to integrate astronomical research and education.

Interest in astronomy has been significantly increased in the country, which also includes amateur astronomy and astrophotography. As a result, the development of astronomical research, education and public outreach are progressing.

\section{Research topics}

Astronomical research in Abastumani Astrophysical Observatory comprises all major fields of astronomy, astrophysics, cosmology and atmospheric sciences, both observational and theoretical studies. It includes solar system bodies, solar physics, stellar and extragalactic astronomy, theoretical astrophysics, cosmology, atmosphere and near space physics.

Below we briefly present the research subjects that the ABAO researchers are working on during last years.

Near-Earth asteroids. ABAO is involved in GAIA and ISON international networks to monitor near-Earth asteroids (NEA). Photometrical and astrometrical observations of about 180 NEAs had been observed during last year. Some possibly hazardous asteroids were monitored. These observations are carried out also in cooperation with Goldstone observatory (USA), simultaneously with radar observations. The observations are done by the 70-cm Meniscus telescope of ABAO.

Selected asteroids are observed to detect YORP effect (Durech et al. 2017). Some asteroids are monitored to detect their duality, among which a few double 
bodies have been detected, including the double asteroid 5872 Sugano (Vokrouhlický et al. 2017). Asteroid clusters are observed and studied recently (Pravec et al. 2018).

These works are carried out in cooperation with Astronomical Institute of the Czech Republic, Chuguevo Observatory of Kharkov State University of Ukraine and many other observatories (see the references above).

Variable stars. Long-term observations of hypergiant stars P Cygni, V509 Cas and EM Cep have been carried out in ABAO for decades. Peculiarities of their variability are studied (Kochiashvili et al. 2018, Michaelis et al. 2018, Kochiashvili et al. 2018). These works are made in cooperation with Jena Observatory (Germany), Ondřejov Observatory (Czhech Republic), Shemakha Observatory (Azerbaijan), Ariel University (Israel).

Extragalactic objects. $A B A O$ is a member of the global network of optical followups of $\gamma$-bursts (Abbott et al. 2017, Volnova et al. 2017) which study variability and nature of the source objects.

ABAO researcher $\mathrm{R}$. Inasaridze co-authored the papers in the journal "Nature" on variations of the nearby black-hole binary V404 Cygni (Kimura et al. 2016) and "Nature Astronomy" on flow transitions of GRB 160625B (Zhang et al. 2018).

Objects with active galactic nuclei (AGN) are studied at various spectral regions using the data of space missions (Fermi, RXTE, Swift, XMM-Newton, Chandra, BeppoSAX, ASKA). AGN variability are investigated during long- and short-term flares, as well as microvariability (Kapanadze et al. 2017, Kapanadze et al. 2017, Kapanadze et al. 2018). Statistical properties and correlations of variations at different spectral ranges are studied (Kapanadze et al. 2017). These works are carried out in cooperation with Osservatorio Astronomico di Brera (Italy) and University of Michigan (USA).

$A B A O$ is involved in an international network for multi-wavelength and multiinstrument observations of BL Lacertae objects (Ahnen et al. 2017). Optical observations are conducted using the $70-\mathrm{cm}$ Meniscus telescope of the observatory.

Solar physics. One of the major fields of $A B A O$ is solar studies. Many different aspects of the Sun are investigated by ABAO researchers, both observationally and theoretically.

Alfvén wave dissipation in the solar chromosphere was investigated by the international scientific group including ABAO researcher Teimuraz Zaqarashvili and 
the results were published in the journal "Nature Physics" this year (Grant et al. 2018).

Dynamics of active regions on the Sun during solar flares and eruptions are studied and long-period oscillations are revieled (Dumbadze et al. 2017). Spectrosopic inversions of $\mathrm{Ca}$ line during various classes of solar flares are investigated (Kuridze et al. 2017). Solar tornadoes and their statistical coupling with flares are studied (Mghebrishvili et al. 2018). Quasi-periodic pulsations of solar flares and their role in estimation of coronal parameters are examined (Philishvili et al. 2017).

Numerical modelling of solar spicules is done for adiabatic and non-adiabatic states (Kuźma et al. 2017). Middle and long-period variations of solar activity - the Reiger periodicity is investigated (Gurgenashvili et al. 2017). Rossby waves and their coupling with solar activity are analysed (Zaqarashvili and Gurgenashvili 2018). Solar magnetic tubes, their effect on solar wind turbulence and its heating is studied (Zhelyazkov et al. 2018). Outer corona dynamics in radio waves are also analyzed (Pylaev et al. 2017).

Many of abovementioned researches are performed in cooperation with institutes in Austria, Belgium, Germany, Great Britain, India, Irland, Italy, Poland, USA and other countries. Observations of solar radiation by radio telescope (Ukraine) are carried out.

Oscillations and waves in solar chromosphere and corona and chromospheric spicules are studied (Khutsishvili et al. 2017). Observations of spectral lines in the chromosphere by height and time are made using the $53-\mathrm{cm}$ solar coronagraph of $A B A O$. Spectrographic and polarimetric observations are carried out to study periodicities of these oscillations.

Statistical studies of differential rotation of coronal holes, rotational velocities and their distribution by latitudes and hemispheres, as well as comparison with photospheric rotational velocities are made. Variations of solar spectrum at different spectral regions are investigated (Bagashvili et al. 2017). Precursors of the coronal hole jets are found in solar bright points (Bagashvili et al. 2018). Rotation of the solar coronal holes are studied (Oghrapishvili et al. 2018).

Theoretical astrophysics. Theoretical studies are devoted to magnetorotational instabilities in accretion disks (Gogichaishvili et al. 2018).

Stability of Keplerian flow around a gravitating center is investigated which is important for dynamics of protoplanetary disks (Poniatowski and Tevzadze 2017). 
Multiwavelength studies of pulsars are carried out in cooperation with institutes in India and Poland (Basu et al. 2018).

Theoretical research in plasma astrophysics are carried out also in the Institute of theoretical physics at Ilia State University studying turbulent fluctuations in astrophysical objects (Gogoberidze et al. 2018).

Cosmology. Different aspects of cosmology are studied by cosmologists in ABAO. Primordial magnetic fields are the subject of research which includes main characteristics of inflational magnetic field evolution (Brandenburg et al. 2017, Kahniashvili et al. 2017), their statistical properties and applications to cosmology (Brandenburg et al. 2018). The dark energy models are studied and their testings are carried out (Avsajanishvili et al. 2017, Avsajanishvili et al. 2018).

Another topic is galaxy distributions, statistical methods for the galaxy survey (Burden et al. 2017), baryon acoustic oscillation features in galaxy bispectrum (Pearson \& Samushia 2018). Spiral galaxies with dark matter in galactic clusters were searched and studied (Borchkhadze et al. 2017).

These studies were performed under the international collaboration with USA, Swetzerland, Armenia and other countries.

Atmospheric and near space physics. Long-term observational and theoretical research of the Earth's atmosphere has been conducted in ABAO. Observations of $\mathrm{OH}$ infrared band of the atmospheric mesopause region by the all-sky imager and the spectrometer (GRIPS-5) are carried out to study dynamical processes and temperature variations in that region of the atmosphere. Total electron content (TEC) measurements in the ionosphere by high-frequensy GNSS receiver are made to study space weather impact on the atmosphere-ionosphere. The data of night airglow intensity are analysed at strong geomagnetic disturbances, variations of airglow caused by tidal motions and atmospheric gravity waves. Interannual variations and long-term trends of ozone, as well as cosmic factors effects on atmospheric parameters are investigated (Didebulidze et al. 2018, Todua et al. 2017). Plasma densities and electron temperatures by ground-based and satellite radio measurements are studied and compared (Lomidze et al. 2018).

In these studies ABAO is collaborating with German Space Agency DLR. as well as a member of international networks of VAO (Virtual Alpine Observatory), ACTRIS (Aerosol, Clouds, and Trace gases), Scientific Committee on Solar Terrestrial Physics (SCOSTEP). 
In most of the researches mentioned above the PhD students are involved.

\section{Observatory instruments}

Currently observations are carried out by $70-\mathrm{cm}$ Maksutov system Meniscus telescope, $48-\mathrm{cm}$ reflector, $30-\mathrm{cm}$ telescope, $53-\mathrm{cm}$ solar coronagraph. Currently the project for instrumental modernization is under consideration, which includes the installation of a modern telescope.

Instruments for atmospheric studies include spectrometer GRIPS-5 to measure $\mathrm{OH}$ bands of the airglow, all-sky imager for observations of the oxygen red line of the mesosphere, high-frequency GNSS receiver to measure total electron content, photometers, aerosol lidar, meteorological station, ozonometer.

\section{Conferences, awards, grants}

In 2017 an international conference in solar physics "Our mysterious Sun: magnetic coupling between solar interior and atmosphere" was held in Tbilisi where the leading scientist in this field had participated.

Researchers and research groups of $A B A O$ are involved in the international projects funded by National Science Foundation and European Commission (FP7, H2020). In 2017 researchers and research groups won the award of Rustaveli Georgian National Science Foundation for the best scientists and scientific groups in Georgia.

\section{Education}

Astronomy becomes more popular in Georgia, so the demand for astronomical education increases. Programs at bachelor, master and doctoral levels in astronomy and astrophysics, as well as atmospheric sciences are offered at llia State University. $\mathrm{PhD}$ programs are available also in Tbilisi State University and Samtskhe-Javakheti State University. Students at master and doctoral levels are able to receive grants to cover study expenses. Doctoral grants also give possibility to get internship in the universities abroad which have been won by some of the $\mathrm{PhD}$ students in astronomy. Foreign students visited Ilia State University and ABAO by Erazmus+ program. International master double degree program in atmospheric sciences will start next year, which includes universities in France, Germany and USA.

In 2018 a joint transdisciplinary international doctoral program between Ilia State University Tbilisi and Georg-August-University of Göttingen (Lehmann-Haupt 
doctoral programme) was launched. Under this project PhD students are doing their research in both universities and obtain double PhD degree. Several PhD students in astronomy and cosmology are involved in this program.

\section{Rehabilitation project}

In 2018, under the financial support of the World Bank and Georgian government, the renovation of Abastumani resort was started. One of the major parts of this project is the rehabilitaion of the infrastructure of the Observatory including its main building. In the renovated spaces the public visitor center and science museum will be established.

\section{Public astronomy in Georgia}

Public outreach had always been one of the important activities of ABAO. Thousands visit the observatory every year from all over the world. They can see the museum and attend lectures. During clear nights the visitors are able to watch visible celestial objects through the telescope. Professional astronomers are involved in the excursions. They also deliver public lectures in schools, libraries and other public places all over the country.

Ilia State University organizes scientific picnics for schools and public every year in which the astronomy part is one of the most attractive and interesting activities. Astronomy is also presented in educational and scientific exhibitions taking place in Georgia.

During recent years the amateur astronomer clubs have been established. They regularly organize star parties, exhibitions and public lectures. Astrophotography and astroart become very popular. Some of Georgian astrophotographers won international prizes for their works. A private observatory near Tbilisi is under construction.

In 19-21 October this year Abastumani Observatory hosted the NASA International Space Apps Challenge organized by Tbilisi Startup Bureau and Ilia State University (https://2018.spaceappschallenge.org/locations/abastumani/). 115 people participated in this contest of creating projects and ideas to tackle challenges on Earth and space offered by the NASA event.

Georgia is a member of the South West Asian Regional Office of the International Astronomical Union's (IAU) project "Astronomy for Development". 
The interest in astronomy is rising significantly in Georgia. International collaboration of Georgian researchers is widening. All these increases perspectives for development and future progress of astronomy in Georgia and the region.

\section{References}

Abbott, B. P. et al (including Inasaridze, R.). 2017, ApJL 848, 2

Ahnen, M. L. et al (including Chigladze R., Kimeridze G., Kurtanidze O., Sigua L.). 2017 A\&A 603, 30

Avsajanishvili, O.; Huang, Y.; Samushia, L.; Kahniashvili, T. 2018, EPJC 78, 9

Avsajanishvili, O.; Samushia, L.; Arkhipova, N. A.; Kahniashvili, T. 2017, A\&AT 30, 1, p. 95-114

Bagashvili, S. R.; Shergelashvili, B. M.; Japaridze, D. R.; Chargeishvili, B. B.; Kosovichev, A. G.; Kukhianidze, V.; Ramishvili, G.; Zaqarashvili, T. V.; Poedts, S.; Khodachenko, M. L.; 2017, A\&A 603, 134

Bagashvili, S. R.; Shergelashvili, Bidzina M.; Japaridze, Darejan R.; Kukhianidze, Vasil; Poedts, Stefaan; Zaqarashvili, Teimuraz V.; Khodachenko, Maxim L.; De Causmaecker, Patrick 2018, ApJL, 855, 2

Basu, R.; Mitra, D.; Melikidze, G. I.; Skrzypczak, A. 2018, MNRAS Advance access Borchkhadze, T., Kogoshvili, N. G., Kalloghlian, A.T. 2017, Astrophysics 60, 305 Brandenburg, A.; Durrer, R.; Kahniashvili, T.; Mandal, S.; Yin, W. W. 2018, JCAP 08 Brandenburg, A.; Kahniashvili, T.; Mandal, S.; Pol, A.R.; Tevzadze, A. G.; Vachaspati, T. 2017, Phys. Rev. D 96, 12

Burden, A. , Padmanabhan, N., Cahn, R.N., White, M. J., Samushia, L. 2017, JCAP 03 Didebulidze, G.; Todua, M.; Didebulidze, T. 2018, 42nd COSPAR Scientific Assembly Dumbadze, G.; Shergelashvili, B. M.; Kukhianidze, V.; Ramishvili, G.; Zaqarashvili, T. V.; Khodachenko, M.; Gurgenashvili, E.; Poedts, S.; De Causmaecker, P. 2017, A\&A 597, A93

Durech, J. et al (including Inasaridze, R. Ya.; Ayvazian, V. R.; Kvaratskhelia, O. I.). 2018, A\&A 609, A86

Gogichaishvili, D.; Mamatsashvili, G.; Horton, W.; Chagelishvili, G. 2018, ApJ 866, 2 Gogoberidze, G.; Voitenko, Y. M.; Machabeli G., 2018, MNRAS 480, 2, p. 1864 Grant, S. D. T., Jess, D. B., Zaqarashvili, T. V. et al. 2018, Nature Physics 14, p. 480-483 Gurgenashvili, E.; Zaqarashvili, T. V.; Kukhianidze, V.; Oliver, R.; Ballester, J. L.; Dikpati, M.; McIntosh, S. W. 2017, ApJ 845, 2

Kahniashvili, T.; Brandenburg, A.; Durrer, R.; Tevzadze, A. G.; Yin, W. 2017, JCAP 12 Kapanadze, B. et al. (including Kapanadze, S.). 2017 MNRAS 469, 1655 
Kapanadze, B. et al. (including Kapanadze, S.). 2018 MNRAS 480, 407

Kapanadze, B. et al. (including Kapanadze, S., Mdzinarishvili, T.). 2017, ApJ 854, 1

Kapanadze, S., Kapanadze, B.; Dorner, D.; Vercellone, S.; Romano, P.; Tabagari, L. 2017, Ap\&SS 362, 10

Khutsishvili, D.; Zaqarashvili, T. V.; Khutsishvili, E.; Kvernadze, T.; Kulidzanishvili, V.; Kakhiani, V.; Sikharulidze, M. 2017, Ap\&SS, 362, 12

Kimura M. et al. (including R. Inasaridze). 2016, Nature 529, 54

Kochiashvili, N.; Beradze, S.; Natsvlishvili, R.; Kochiashvili, I.; Vardosanidze, M.; Pannicke, A. 2018, Astrophysics 61, 22

Kochiashvili, N.; Natsvilishvili, R.; Kochiashvili, I.; Vardosanidze, M.; Beradze, S.; Pannicke, A. 2018, Astronomy \& Astrophysics (CAUCASUS), 3

Kuridze, D. et al (including Zaqarashvili, T. V.) 2017, ApJ, 846, 1

Kuźma, B.; Murawski, K.; Zaqarashvili, T. V.; Konkol, P.; Mignone, A. 2017, A\&A 597, 8 Lomidze L., Knudsen D.J., Burchill J., Kouznetsov A., Buchert S.C. 2018, Radio Science 53,1

Mghebrishvili, I.; Zaqarashvili, T.V.; Kukhianidze, V.; Kuridze, D.; Tsiklauri, D.; Shergelashvili, B. M.; Poedts, S. 2018, ApJ, 861, 2

Michaelis, A. M.; Kashi, A.; Kochiashvili, N. 2018, New Astronomy 65, p. 29

Oghrapishvili, N. B.; Bagashvili, S. R.; Maghradze, D. A.; Gachechiladze, T. Z.; Japaridze, D. R.; Shergelashvili, B. M.; Mdzinarishvili, T. G.; Chargeishvili, B. B. 2018, ASR 61, 12 Pearson, D. W.; Samushia, L. 2018, MNRAS 478, 4500

Philishvili, E.; Shergelashvili, B. M.; Zaqarashvili, T. V.; Kukhianidze, V.; Ramishvili, G.; Khodachenko, M.; Poedts, S.; De Causmaecker, P. 2017, A\&A, 600, 12

Poniatowski, L. G.; Tevzadze, A. G. 2017, Phys. Rev. E, 96, 1

Pravec, P. et al. (including Inasaridze, R. Ya.; Ayvazian, V. R.; Kvaratskhelia, O. I.; Zhuzhunadze). 2018, Icarus 304, 110

Pylaev, O. S.; Zaqarashvili, T. V.; Brazhenko, A. I.; Melnik, V. N.; Hanslmeier, A.; Panchenko, M. 2017, A\&A, 601, 6

Todua M., Didebulidze G.G. Javakhishvili G.Sh. 2017, Non-Stable Universe: Energetic Resources, Activity Phenomena and Evolutionary Processes, ASP Conference Series. Astronomical Society of the Pacific

Vokrouhlický, D. et al. (including Inasaridze, R. Ya.; Ayvazian, V. R.) 2017, AJ, 153, 6

Volnova, A. A. et al. (including Inasaridze, R. Ya. 2017, MNRAS, 467, 3500

Zaqarashvili, T. V.; Gurgenashvili, E. 2018, Ap\&SS, 5, 7

Zhang, B.-B. et al. (including R. Inasaridze). 2018, Nature Astronomy 2, 69

Zhelyazkov, I.; Zaqarashvili, T. V.; Ofman, L.; Chandra, R. 2018, ASR 61, 628 